\title{
Implementation of Medical Behavior Management System in Operating Rooms
}

\author{
Guimei Zhang1, Yingzi Yuan²* \\ ${ }^{1}$ Xiang'an Hospital of Xiamen University, Xiamen 361000, Fujian Province, China \\ ${ }^{2}$ Harbin Medical University Cancer Hospital, Harbin 150040, Heilongjiang Province, China \\ *Corresponding author: Yingzi Yuan, yuanyingzi2008@163.com
}

\begin{abstract}
Objective: To strengthen personnel management for a clean operating room and ensure an automatic, intelligent, and scientific workflow. Methods: The medical behavior management system has been implemented to monitor and manage medical personnel entering and exiting the operating room, so as to meet the standard requirements of the operating room. Results: The flow of personnel has been controlled effectively, the flow in and out of the operating room has been optimized, the management level of the operating room has improved, and the cost has been cut down. Conclusion: With the advent of the information age and the continuous improvement of the management system, the management of operating rooms has become more reasonable and humanized; the management mode, working environment, and the overall quality of nursing work in operating rooms have improved.
\end{abstract}

Keywords: Medical behavior management system; Personnel management; Medical personnel; Auxiliary personnel

Publication date: November 2021; Online publication: November 30, 2021

\section{Introduction}

With the rapid development of surgery, it is an inevitable need for the development of the times to provide a clean and safe operating environment for patients with the use of information management. The behavior management of operating room personnel mainly includes the behavior management of surgeons, anesthesiologists, operating room nurses, and other personnel entering the operating room. By managing the pathway, hand-washing clothes, operating shoes, and articles of personnel entering the operating room according to national norms, this would ensure that operating personnel would meet the requirements and access standards before entering the operating room.

At present, the behavior management of most hospitals is mainly through the manual management of auxiliary personnel. Although it is a standard that operating personnel should ensure their cleanliness before entering the operating room, the phenomena whereby the management efficiency is low, the dominance of human relationship over management, and the recurring mistakes in the management process still occur due to a weak sense of responsibility among auxiliary personnel as they do not dare or are unwilling to offend people. Therefore, the cleanliness requirements of the operating room cannot be guaranteed. Due to the medical behavior management of auxiliary personnel management, hand-washing clothes are distributed to different personnel after each disinfection; it is impossible to trace back the original user and return the hand-washing clothes. If hand-washing clothes are not returned after surgery, they cannot be found in time, leading to the loss of resources and the hospital. Auxiliary personnel usually manage the operating personnel in terms of wearing hand-washing clothes and operating shoes while entering and exiting the operating room; however, in the operating room, they cannot effectively control the operating personnel's behavior, which may result in the pollution of hand-washing clothes and operating shoes. In 
view of that, intraoperative infection may easily occur. With the continuous development of information technology, its use in managing the behavior of operating room personnel has played a very important role in the management of operating rooms.

\section{General information}

Harbin Medical University Cancer Hospital is a Grade-III Class-A cancer hospital. Since 2016, the operating room in the new outpatient complex building covers an area of $7000 \mathrm{~m}^{2}$, and the area that is used is nearly $5000 \mathrm{~m}^{2}$. A total of 27 new operating rooms have been built, with an annual operation volume of more than 15,000 cases, an average of 80-100 cases per day, and 300-500 people entering and leaving the operating room every day. In order to ensure a normal operation and avoid the occurrence of cross infection, a scientific, reasonable, and effective maintenance of the property of the hospital and departments is necessary; thus, scientific management has been adopted, the medical behavior management system has been introduced, and information technology is being used to manage the behavior of medical personnel. This system includes access control system, intelligent dispensing machines for clothes, shoe cabinets, wardrobes, and clothes receiving machine.

\section{Management of the medical staffs}

\subsection{Registration of personnel}

This includes recording the information of surgical, anesthesiology, and operating room personnel who need to enter the operating room in advance, entering the information into the system, and registering them. According to the registration information, identity cards would be issued to relevant medical staffs recognized by the system to gain access to the operating room, swipe their card to take clothes, open shoe cabinets, return clothes, and other functions.

\subsection{Management of hand-washing clothes}

The operating room is equipped with electronic shoe cabinets and dressing cabinets, which can be set by the operating personnel through the system. Fixed shoe cabinets and wardrobes should be provided for anesthesiology and operating room staffs, while temporary shoe cabinets and wardrobes should be provided for surgeons, refresher doctors, and students. When an operating personnel leaves the operating room, the system would automatically delete the occupational relationship of the shoe cabinets and dressing cabinets that have been used.

\subsection{Recycling of hand-washing clothes}

After surgery, the staffs would use their identity cards to open the wardrobes and shoe cabinets automatically. Before leaving the dressing room, the staffs should place the hand-washing clothes in the automatic recycling machine and swipe their cards to automatically recycle those clothes. If the handwashing clothes are not returned for 24 hours, the display screen would continue to scroll.

\subsection{Entering the operating room}

Staffs would swipe their cards to gain access. Then, they would go to the clothes dispensing machine to take the hand-washing clothes and slippers to change their clothes and shoes. Walking through the office area, they would then enter the clean operation department. 


\subsection{Exiting the operating room}

After surgery, medical staffs would go to the dressing room to change their clothes, swipe their cards to return those clothes, change their shoes, and exit in reverse, making the workflow more intelligent.

\section{Problems encountered during the implementation and their countermeasures}

(1) The clothes dispensing machine has added the function of card-swiping, in order to take the second clothing (long-sleeved clothing). After taking the first hand-washing clothing, the second clothing can be taken according to individual needs.

(2) Set the waiting time from the card-swiping to taking the first and second clothing. Provide voice prompt to ensure that the second clothing would not be taken before the card-swiping of the first clothing, so as to avoid a time interval that is too short, which would result in the clothes not being taken out. After swiping the card to take the first clothing, wait a few seconds before swiping the card again to take the second clothing.

(3) In regard to the clothes receiving machine, upon reaching a certain number of clothes (30 clothes), the personnel would no longer be able to return the clothes, and the program would prompt to contact the administrator. It is necessary to notify the front desk staff to replace the clothes receiving bag in the machine, in order to continue its function.

(4) Add the classification of clothes size in the program of the clothes receiving machine and display the quantity of clothes of each size along with the sleeve size on the program interface for convenience.

(5) When placing in clothes into the clothes dispensing machine, if the clothes have not been returned, there would be a voice prompt directing the user to return the clothes first and then add clothes to the machine.

(6) Add the return function on the client program. If an individual adds clothes to the clothes dispensing machine, the voice prompt for unreturned clothes would appear; the individual can then return the clothes and add clothes to the clothes dispensing machine.

(7) Modify the large screen program to only display the information of doctors and nurses who would be swiping their cards to take clothes and open the cabinet on that day and the information of surgeons and nurses who would swipe their cards to take clothes and return clothes on that day in real time and dynamically.

(8) Modify the large screen program in the operating room to prevent it from shutting down automatically. Run the large screen program for a long time and 24 hours a day to display medical information.

(9) Add a pop-up window on the client to display the medical information of the clothes that have not been returned for 24 hours, so that the front desk staff can identify the medical personnel who should be held accountable.

(10) Add the remaining number of clothes of various sizes in the clothes dispensing machine system to assist the front desk staff in determining when to add the clothes based on the remaining number of clothes in the machine.

(11) Display the remaining number of clothes of each size in the clothes dispensing machine on the client to save time and effort as the staff do not need to go to the clothes dispensing machine to check but instead from the client itself. 
(12) Add the total number of returned clothes in the clothes receiving machine and check the total number of clothes.

(13) Modify the system where the door cannot be opened by swiping the card.

(14) Add the function of No. 5 clothes to the clothes receiving machine and client program. Staffs can then swipe their card to take the clothing from the clothes dispensing machine.

(15) Modify the position of the remaining clothes of various sizes in the clothes dispensing machine on the client program interface, move it to the blank part on the right of the program, enlarge the font, and bold it. Ensure that the modification is clear and obvious for viewing.

(16) Add a typesetting function to the clothes dispensing machine. When medical personnel swipe their cards to take the clothes from the clothes dispensing machine, voice broadcast the number of ongoing operations and the corresponding operating rooms.

(17) Add the information about the remaining space in the wardrobes and shoe cabinets on the client and display them by category to facilitate and quickly check the usage of the wardrobes and shoe cabinets.

(18) Modify the function of the conversation system, remove the function of displaying videos in the operating room, and only display medical records. Add the patient's signature function. After modification, staffs can then view the medical records, and patients can sign on the anesthesia consent form.

(19) Add a large screen information query and personal information query on the client to facilitate the staffs to view the information of the medical staffs who have taken the clothes and returned them.

(20) At the bottom right of the client main interface, it would display a signal when the clothes and shoes cabinets are not closed, reminding the receptionist to close the door to avoid misplacing the clothes and shoes. This is convenient for the staffs in reminding them to close the cabinet door.

\section{Conclusion}

There are many disadvantages in the behavior of medical personnel under the simple management of auxiliary personnel. The purpose of effectively controlling the cleanliness of the air in the operating room cannot be achieved by relying on the management of people and system. By applying the medical behavior management system to the management and control of the operating room as well as combining the access control system, electronic locker, shoe cabinet, intelligent dispensing machine for clothes, clothes receiving machine, and information publicity screen, it is possible to strictly and effectively control the access standards of personnel entering the operating room as well as strengthen the effective management of the medical environment and order in the operating room, thus reducing the risk of postoperative infection. The medical behavior management system realizes the intelligent distribution and recycling of hand-washing clothes as well as the automatic distribution of dressing cabinets and shoe cabinets. Within one year of operation, the problems existing in the use of the medical behavior management system and the causes have been analyzed, appropriate solutions have been suggested, and strict operation procedures have been formulated. It does not only optimize the management process of the dressing area and shoe changing area as well as effectively control the personnel flow, but also provide information-based management means to reduce the workload and labor cost. With the continuous rise of the operation volume and the improvement of the management system, the management of operating rooms needs to be more reasonable, intelligent, and humanized. 


\section{Disclosure statement}

The authors declare that there is no conflict of interest.

\section{References}

[1] Zhao T, Li X, 2012, Development and Application of Digital Operating Room Safety Door Control Management System. Chinese Journal of Nursing, 47(11): 3.

[2] Yao G, Zhang X, Wang H, 2013, Behavior Management System Design of Digital Operating Room. China Digital Medicine, 2013(11): 4.

[3] Wang Y, 2011, Infection Control and Equipment Management in Modern Operating Room. Medical \& Pharmaceutical Journal of Chinese People's Liberation Army, 23(2): 101-103.

[4] Huang D, Liu J, Zhao Y, 2010, Application of Critical Control Point Method in Clean Operating Room. Chinese Journal of Disinfection, 27(5): 2.

[5] Li F, Guo L, Hu P, 2006, Experience of Operation Staff Management in Clean Operating Room. Journal of Clinical Nursing, 2006(1): 2.

[6] Yang Y, Yin L, Li H, et al., 2016, Design and Application Practice of Behavior Management System of Intelligent Clothes and Shoes in Operating Room. China Medical Device Information, 2016(14): 4. 